\title{
Keine «Rationierung» im geltenden KVG
}

Bemerkungen zu BGE 145 V 116

Thomas Gächter *

Spätestens seit der viel diskutierten «Erwägung 7» des Myozyme-Entscheids von 2010 (BGE 136 V 395) stellte sich die Frage, ob im Rahmen des krankenversicherungsrechtlichen Wirtschaftlichkeitsgebots eine Obergrenze für die Kostenübernahme bestehe. Im Fall eines 71-jährigen Patienten, der aufgrund verschiedener Komplikationen Spitalkosten von über zwei Millionen Franken verursacht hatte, hat das Bundesgericht dies nun mit aller wünschbaren Klarheit verneint.

I. Sachverhalt und Ausgangslage ...............................................................207

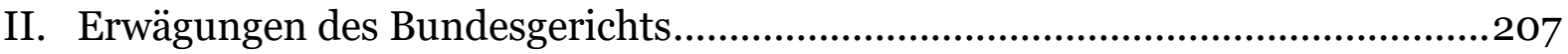

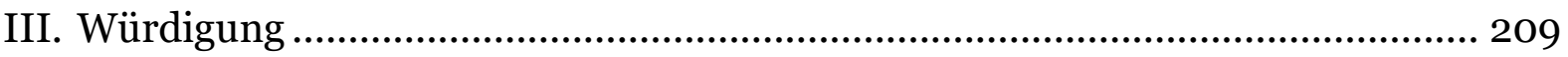

1. «Keine absolute Obergrenze für die Übernahme der Kosten einer

Spitalbehandlung» .................................................................................... 209

2. QALY-Konzept und Gesamtkostenbetrachtungen ..................................211

3. Verantwortlichkeit des Gesetzgebers ......................................................211

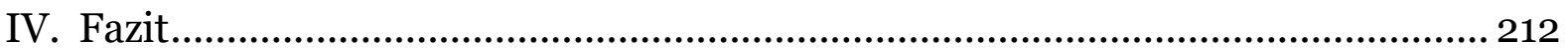

Zitiervorschlag: Thomas Gächter, Keine «Rationierung» im geltenden KVG, in: sui-generis 2019, S. 206

URL: $\quad$ sui-generis.ch/103

DOI: $\quad$ https://doi.org/10.21257/sg.103

* Prof. Dr. iur. Thomas Gächter (thomas.gaechter[at]rwi.uzh.ch), Ordinarius für Staats-, Verwaltungs- und Sozialversicherungsrecht an der Universität Zürich. 


\section{Sachverhalt und Ausgangslage}

1 Ein damals 71-jähriger Versicherter trat 2014 für eine Knieoperation in ein Spital ein. Nach dem Eingriff erlitt er zunächst einen Herzinfarkt, danach ein Nierenversagen und anschliessend zahlreiche weitere Komplikationen, die jeweils entsprechend behandelt wurden. Mehrere dieser Komplikationen waren akut lebensbedrohlich. Erst nach 421 Tagen Behandlung, wovon ein wesentlicher Teil auf der Intensivstation stattfand, wurde der Versicherte aus dem Spital entlassen. Rund zwei Monate nach der Entlassung verstarb der Versicherte aufgrund erneuter gesundheitlicher Komplikationen.

Der Gesamtbetrag der Behandlungskosten belief sich auf rund 2,4 Millionen Franken; 1,08 Millionen Franken davon forderte das Spital vom Krankenversicherer des Versicherten. Dieser übernahm allerdings nur 300'00o Franken. Er begründete dies damit, dass nach seinen Berechnungen nicht mehr geschuldet sei. Auf Klage des Spitals verpflichtete das Schiedsgericht in Sozialversicherungssachen des Kantons Basel-Stadt den Krankenversicherer 2018 zur Bezahlung des Differenzbetrages, wogegen sich dieser erfolglos vor Bundesgericht wehrte.

\section{Erwägungen des Bundesgerichts}

3 Das Bundesgericht handelte zunächst die allgemeinen Leistungsvoraussetzungen der obligatorischen Krankenpflegeversicherung ab (E. 3). Dies ist im Gesamtzusammenhang des Entscheids von erheblicher Bedeutung, weil das Gericht seinen konkreten Entscheid letztlich exakt in die allgemeinen und gesetzlich fixierten Leistungsvoraussetzungen und Leistungseinschränkungen einpasst und der Geltung aussergesetzlicher Leistungsbeschränkungen eine Absage erteilt.

4 Insbesondere im Zusammenhang mit der Leistungsvoraussetzung der Wirtschaftlichkeit im Sinne von Art. 32 KVG betont das Bundesgericht, dass es sich bei dieser um einen vergleichenden Massstab zwischen mehreren, ihrerseits wirksamen und zweckmässigen Behandlungsalternativen handle, der die Wahl der wirtschaftlicheren Alternative gebiete. "Die Frage der Wirtschaftlichkeit stellt sich grundsätzlich nicht, wenn es nur eine Behandlungsmöglichkeit bzw. keine Behandlungsalternative gibt, weil sich das in Art. 32 Abs. 1 KVG verankerte Erfordernis auf die Wahl unter mehreren zweckmässigen Behandlungsalternativen bezieht» (E. 3.2.3).

5 Der Krankenversicherer stellte sich dagegen auf den Standpunkt, dass der konkrete Fall gar in mehrfacher Hinsicht Ausnahmecharakter aufweise. So seien im konkreten Fall neunundsechzig Mal höhere Kosten angefallen, als dies mit rund $35^{\circ} \mathrm{OOoo}$ Franken nach neueren Studien bei Spitalaufenthalten in den letzten zwölf Lebensmonaten durchschnittlich der Fall gewesen sei. Da das Bundesgericht sich in BGE $136 \mathrm{~V} 395$ auf die sogenannte QALY-Methode beziehe (d.h. eine Methode, in welcher die mit einer Behandlung gewonnenen quality-adjusted life years berechnet werden) und das Bundesgericht zum Ausdruck gebracht habe, dass pro QALY rund 100'00o Franken an Kosten der Krankenpflegeversicherung angemessen wären, ergebe sich im konkreten Fall ein QALY-Wert von 2.96, was durch die von der Versicherung vergüteten 100'00o Franken zum Ausdruck gebracht werde. Die Berechnung des QALY- 
Wertes stützte sich auf die statistische Rest-Lebenserwartung des 71-Jährigen (14.8 Jahre), die mit einem Lebensqualitätswert von 0.2 (entsprechend dem Umstand, dass der Versicherte in allen Verrichtungen des Lebens massiv beeinträchtigt oder behindert gewesen wäre) multipliziert wurde. Die vom Bundesgericht im Zusammenhang mit Arzneimitteln (BGE 136 V 395) und SpitexLeistungen (BGE 142 V 144) entwickelten Überlegungen sind nach Ansicht des Krankenversicherers auch auf Spitalaufenthalte anzuwenden. Es handle sich dabei um eine «lückenfüllende Rechtsprechung», die Gesetzesrang habe und sich auf das Gebot der Verhältnismässigkeit stütze, das «eine absolute Grenzziehung» verlange (E. 4.2). Dagegen führte das Spital an, dass gerade für eine solche, separate und gesetzlich nicht vorgesehene Verhältnismässigkeitsprüfung durch ein Gericht kein Raum bestehe, zumal das Bundesgericht eine Kostenobergrenze von 100'00o Franken pro QALY nie verbindlich festgehalten habe (E. 4.3).

Das Bundesgericht stellte sich in der Folge klar gegen die Rechtsauffassung der Krankenversicherung und verneinte insbesondere, dass sich aus den wichtigsten angeführten Präjudizien (BGE 136 V 395; BGE 142 V 144; BGE 142 V 478) entsprechend Grundsätze ableiten liessen (E. 5.3):

- In BGE 136 V 395 (Myozyme) habe das Bundesgericht zwar in der Tat das gesundheitsökonomische QALYKonzept diskutiert, vor allem um aufzuzeigen, dass in anderen Ländern die Verhältnismässigkeit von Leistungen mit Hilfe dieses Konzept bemessen werde. Allerdings habe sich der konkrete Entscheid nicht auf dieses Konzept abgestützt.

- In BGE 142 V 478 (Myozyme II) waren die Kosten des unterdessen (mit stark reduzierten Preisen) auf der Spezialitätenliste figurierenden Medikaments zu beurteilen. Diese wurden allein schon deshalb als wirtschaftlich betrachtet, weil die Aufnahme auf die Spezialitätenliste mit Limitierungen und einer wirtschaftlich geprüften Preisfestsetzung verbunden sei und insofern für eine weitere Wirtschaftlichkeitsprüfung kein Raum verbleibe.

- Schliesslich sei in BGE 142 V 144, in dem Überwachungsmassnahmen im Umfang von rund 200`ooo Franken pro Jahr für eine Versicherte mit Undine-Syndrom beurteilt wurden, zwar auch nicht ausdrücklich ausgeschlossen worden, dass bei Alternativlosigkeit einer Behandlung gegebenenfalls unter dem Aspekt der Verhältnismässigkeit eine Leistung verweigert werden könnte, doch hätten diese Überlegungen dann im Entscheid selbst keine tragende Rolle gespielt.

7 Das Bundesgericht hielt im Ergebnis fest, dass in den genannten Entscheiden zwar von bestimmten gesundheitsökonomischen Zahlen die Rede gewesen sei, jedoch nie eine absolute Grenze für die zulasten der obligatorischen Krankenpflegeversicherung gehenden Kosten festgelegt worden sei (E. 5.4).

8 Wichtig ist sodann der in Erwägung 6 des Entscheids besprochene Aspekt: Im Unterschied zu den soeben beschriebenen Entscheiden steht im konkreten Fall 
nicht die Wirtschaftlichkeit einer einzelnen Massnahme zur Diskussion. Vielmehr beanstandet die Krankenversicherung pauschal den nach einer Vielzahl von medizinischen Vorkehren aufgelaufenen Gesamtbetrag. Hierzu hält das Gericht deutlich fest, dass die Kritik der Krankenversicherung von vornherein nur zielführend sein könnte, wenn sie bei den einzelnen Massnahmen ansetzen würde. Im vorliegenden Fall fehlten allerdings Anhaltspunkte dafür, dass einzelne durchgeführte Massnahmen nicht wirtschaftlich gewesen wären (E. 6.2).

9 Zum Argument, dass die Gesamtkosten hier neunundsechzig Mal höher gewesen seien als die durchschnittlich anfallenden Spitalkosten im letzten Lebensjahr von 35‘ooo Franken, stellt das Gericht klar, dass eine unbeschränkte Leistungspflicht der obligatorischen Krankenpflegeversicherung besteht, solange die im Rahmen der Spitalbehandlung vorgenommenen einzelnen Massnahmen die Voraussetzungen der Wirksamkeit, der Zweckmässigkeit und der Wirtschaftlichkeit erfüllen. Anzeichen, dass es an einer dieser Voraussetzungen gefehlt hätte, erkennt das Gericht nicht (E. 6.3).

Entsprechend diesen Ausführungen anerkennt das Bundesgericht den geltend gemachten Aufwand des Spitals als im Rahmen der Leistungsvoraussetzungen der Krankenversicherung vollumfänglich gerechtfertigt und verpflichtet die Krankenversicherung, den noch ausstehenden Betrag zu vergüten (E. 6.4).

\section{Würdigung}

\section{1. «Keine absolute Obergrenze für die Übernahme der Kosten einer Spitalbehandlung»}

11 Die Medienmitteilung des Bundesgerichts vom 23. April 2019 zu diesem Entscheid war überschrieben mit «Obligatorische Krankenpflegeversicherung: Keine absolute Obergrenze für die Übernahme der Kosten einer Spitalbehandlung». ${ }^{1}$ Damit ist die wichtigste Kernaussage des Entscheids treffend zusammengefasst.

12 Allerdings erschliesst sich der eigentliche Sinn der Aussage erst, wenn man den aufgrund des Wortlauts und der Systematik des Gesetzes nicht überraschenden - Entscheid in den weiteren Kontext stellt. Diesen Kontext bildet der viel diskutierte BGE 136 V 395 (Myozyme), namentlich dessen Erwägung 7. In dieser wurde erstmals ausdrücklich und ausführlich in einem bundesgerichtlichen Urteil die Möglichkeit der Leistungsverweigerung aus wirtschaftlichen Gründen diskutiert. ${ }^{2}$ Das Bundesgericht hatte im

\footnotetext{
Medienmitteilung des Bundesgerichts vom 23. April 2019 zum Urteil vom 1. April 2019 (9C_744/2019), Korrespondenznummer 11.5.2/16_2019.

2 Zur damaligen Diskussion des Entscheids etwa Tomas Poledna/Marianne Tschopp, Der Myozyme-Entscheid des Bundesgerichts. Ethik, Rationierung und Grenzen der Finanzierung des Gesundheitssystems - ein Meilenstein für die Rechtsprechung und das Gesundheitsrecht, in: Jusletter vom 7. Februar 2011 (www.jusletter.ch; = Schweizerische Zeitschrift für Gesundheitsrecht RSDS/SZG 2012, $377 \mathrm{ff}$.). Siehe auch Thomas Gächter/Arlette Meienberger, BGE 136 V 395 (Morbus Pompe / Myozyme). Was der Entscheid bedeutet - und was nicht, in: Haftung und Versicherung (HAVE) 2/2013, S. 168-170; Valérie Junod/Jean-Blaise Wasserfallen, Rationnement des soins: le TF jou enfin cartes sur table, Commentaire de l'ATF $136 \mathrm{~V} 395$, in: Jusletter vom 29. August 2011 (www.jusletter.ch; = Schweizerische Zeitschrift für Gesundheitsrecht RSDS/SZG 2012, 223 ff.).
} 
konkreten Fall einer Patientin, die an der seltenen Erbkrankheit Morbus Pompe litt, die (weitere) Vergütung der Behandlung mit dem extrem teuren Arzneimittel Myozyme verweigert. Das (damals) ${ }^{3}$ nicht auf der Spezialitätenliste geführte Medikament war im vereinfachten Verfahren nach Art. 14 HMG als wichtiges Medikament für seltene Krankheiten (orphan drug) von Swissmedic zugelassen worden. Nach der damaligen Rechtsprechung des Bundesgerichts, 4 die per 1. März 2011 in Verordnungsrecht überführt wurde, 5 sind die Kosten für ein nicht auf der Spezialitätenliste geführtes Arzneimittel ausnahmsweise vergütungspflichtig, "wenn ein sogenannter Behandlungskomplex vorliegt oder wenn für eine Krankheit, die für die versicherte Person tödlich verlaufen oder schwere und chronische gesundheitliche Probleme nach sich ziehen kann, wegen fehlender therapeutischer Alternativen keine andere wirksame Behandlungsmethode verfügbar ist; diesfalls muss das Arzneimittel einen hohen therapeutischen (kurativen oder palliativen) Nutzen haben». ${ }^{6}$

$3 \longdiv { \text { In BGE } 1 4 2 \text { V } 4 7 8 \text { behandelte das Bundesgericht } }$ dann einen Fall, in dem Myozyme zur Anwendung kam, nachdem es auf die Spezialitätenliste aufgenommen worden war.

4 Siehe BGE 136 V 395, E. 5.2, mit weiteren Hinweisen.

5 Art. 71a ff. KVV.

6 Eingehend zur Entwicklung dieser Fragestellung Loris Magistrini, L'utilisation hors étiquette de médicament et son remboursement par l'assurance-maladie, in: Jusletter vom 31. Januar 2011 (www.jusletter.ch), N. 65 ff. (= Schweizerische Zeitschrift für Gesundheitsrecht RSDS/SZG 2012, $299 \mathrm{ff}$.), und zur Bedeutung von Art. 71a und $71 \mathrm{~b}$ KVV derselbe Autor, Remboursement de médicaments par l'assurance obligatoire des soins: Commentaire des nouveaux arts. $71 \mathrm{a}$ et $71 \mathrm{~b}$ OAMal, in: Jusletter vom 30. Januar 2012 (www.jusletter.ch; = Schweizerische Zeitschrift für Gesundheitsrecht RSDS/SZG 2012, 95 ff.).
13 Das Bundesgericht verneinte im konkreten Fall das Vorliegen eines hohen therapeutischen Nutzens, da das Ausmass der durch die Behandlung erreichten Verbesserungen ungewiss war und weder mit allgemeinen klinischen Studien noch im konkreten Fall verlässlich nachgewiesen werden konnte. 7 Damit wäre der Fall erledigt gewesen. Das Gericht aber behandelte darüber hinaus in der bereits erwähnten Erwägung 7 ausführlich, und ohne konkrete Anwendung im Einzelfall, die Frage des Kosten-Nutzen-Verhältnisses und der Rechtsgleichheit im Hinblick auf die Grenzen der Finanzierbarkeit der Gesundheitsversorgung. ${ }^{8}$ Im Ergebnis werden in der Literatur, laut Bundesgericht, Beträge in der Grössenordnung von rund 100'ooo Franken pro gerettetes Menschenlebensjahr - wobei sich die verschiedenen vom Bundesgericht herangezogenen Zahlen nicht so genau eingrenzen lassen und keineswegs zwingend von 100'0oo Franken die Rede war - noch als angemessen betrachtet. Die Behandlung mit Myozyme wäre deshalb nach Ansicht des Bundesgerichts im Lichte dieser Meinungsäusserungen auch bei einem nachgewiesenen hohen therapeutischen Nutzen nicht vergütungspflichtig. 9

14 Vor diesem Hintergrund ist nun ersichtlich, weshalb sich die Frage nach einer «absoluten Obergrenze» überhaupt stellte - und weshalb sich die Zahlungsbereitschaft des Krankenversicherers auf rund $100^{\prime}$ ooo Franken pro vermutlich «gerettetes» Lebensjahr beschränkte. Aus dieser Perspektive erschliesst sich

\footnotetext{
BGE 136 V 395, E. 6.10.

8 Das Bundesgericht bezeichnete im besprochenen Entscheid diese Erwägung 7 denn auch ausdrücklich als obiter dictum (BGE 145 V 116 E. 5.4).

9 Siehe BGE 136 V 395, E. 7.8.
} 
nun auch die eigentliche Tragweite des Entscheides: Er klärt, dass es - trotz anderslautenden Erwägungen in BGE 136 V 395 E. 7 - im Rahmen des geltenden Krankenversicherungsrechts keine wirtschaftliche Leistungsobergrenze gibt, wenn die einzelnen Leistungen sachlich und medizinisch begründet sind, selbst wenn sie sich zu sehr hohen Beträgen kumulieren. «Wirtschaftlichkeit» i.S.v. Art. 32 KVG ermächtigt die Versicherer damit gerade nicht zur Verweigerung von teuren Leistungen im Einzelfall, auf die aufgrund des übrigen Leistungsrechts Anspruch besteht. Eine Leistungsverweigerung aus wirtschaftlichen Gründen ist nur zulässig, wenn eine (günstigere) Behandlungsalternative mit vergleichbarem Nutzen zur Verfügung steht. ${ }^{10}$

\section{QALY-Konzept und} Gesamtkostenbetrachtungen

Zwei Begründungslinien, die von der Beschwerdeführerin geltend gemacht werden, verwirft das Bundesgericht ebenfalls deutlich:

Einerseits stellt es klar, dass das gesundheitsökonomische QALY-Konzept in bundesgerichtlichen Entscheiden wiederholt diskutiert worden ist, ohne dass dieses als Teil der anwendbaren Grundsätze anerkannt worden oder gar ein bestimmter Höchstbetrag pro QALY definiert worden wäre. Die immer wieder genannten - und von der Krankenversicherung im konkreten Fall angewandten - 100'00o Franken pro QALY finden je-

$10 \overline{\text { So auch Gächter/Meienberger (Fn. 2), } 169 \text { f.; so }}$ nun auch klar im hier besprochenen Entscheid (BGE 145 V 116 E. 3.2.3). denfalls weder in Gesetz noch Rechtsprechung ${ }^{11}$ eine Grundlage.

17 Anderseits verwahrt sich das Gericht gegen die vom Krankenversicherer praktizierte Gesamtkostenbetrachtung: Im System der obligatorischen Krankenpflegeversicherung, das auf die Vergütung einzelner Leistungen (nach verschiedenen Tarifmodellen) ausgelegt ist, können nicht die addierten Leistungen einer versicherten Person massgeblich sein, wenn es um die Prüfung der Wirtschaftlichkeit geht. Es steht immer nur die jeweilige (einzelne) Leistung zur Überprüfung. Eine Gesamtobergrenze für die Übernahme kumulierter Leistungen besteht nach dem geltenden System demnach nicht.

\section{Verantwortlichkeit des Gesetzgebers}

18 Mit dem hier besprochenen Entscheid hat das Gericht die Frage der hohen Kosten im Krankenversicherungsrecht nicht gelöst, sondern lediglich klargestellt, dass es nicht in der Kompetenz der Versicherer oder der Gerichte liegt, gestützt auf Wirtschaftlichkeits- oder Verhältnismässigkeitsüberlegungen die Vergütung sachlich gerechtfertigter Krankenpflegeleistungen zu verweigern.

19 Solche Überlegungen, die sich mit der Zuteilung von Ressourcen im Einzelfall beschäftigen, müssen auf einer höheren, demokratisch legitimierten Ebene angestellt werden. Nicht die Rechtsanwendung, sondern der Gesetzgeber steht in der Pflicht, die entsprechenden Weichen zu stellen. Der Konsens darüber, welche Kosten bei welchem Nutzen noch akzep-

11 Und auch nicht in der bisherigen Versicherungsund Gerichtspraxis, die weit höhere Beträge bereits heute vergütet. 
tabel erscheinen, wie der Nutzen bewertet werden kann, wo die Grenzen der Finanzierbarkeit liegen und wie die vorhandenen Mittel gerecht verteilt werden, muss in politischen Auseinandersetzungen gefunden und in Gesetzen, Verordnungen und Listen normiert werden. ${ }^{12}$ «Die von der Rechtsgleichheit geforderte Angemessenheit der Leistungsdifferenzierung kann nicht vom Richter durch einzelfallmässige Entscheidung von Fragen mit sozialpolitischem und gesamtwirtschaftlichem Charakter erfolgen.»13

20 Wirtschaftlichkeitsüberlegungen im Einzelfall, die $\mathrm{zu}$ einer Leistungsverweigerung führen, sind demnach nur zulässig, wenn die Rahmenbedingungen hierfür auf höherer Ebene definiert worden sind. In der konkreten Rechtsanwendungssituation wurden alle Leistungen sachgerecht und $\mathrm{zu}$ Recht erbracht und findet sich keine gesetzliche Obergrenze. Es ist $\mathrm{zu}$ begrüssen, dass das Bundesgericht dies mit aller Klarheit festgestellt hat; nun ist es am Gesetzgeber zu handeln, wenn er - was mir nicht zwingend erscheint - einen entsprechenden Handlungsbedarf sieht.

\section{Fazit}

21 Nach den sehr allgemein gehaltenen und über den Einzelentscheid hinausgehenden Ausführungen der Erwägung 7 von BGE 136 V 395 war es eine Frage der Zeit, bis die Rechtsprechung zur Tragweite dieser Überlegungen Stellung nehmen musste. Der vorliegende Fall mit seinen sehr hohen Kosten und den pointierten Stellungnahmen des Krankenversiche-

12 Gächter/Meienberger(Fn. 2), 38.

13 Jörg Paul Müller, Soziale Grundrechte in der Verfassung, 2. Aufl., Basel 1981, 197. rers bot die nahezu ideale Gelegenheit hierfür.

22 Die bundesgerichtliche Klärung ist sehr deutlich ausgefallen. Das Gericht hat sich zwar nicht ausdrücklich von der Ausnahmeoption der Leistungsverweigerung wegen $\mathrm{zu}$ hoher Kosten im Einzelfall (d.h. bei einer Einzelleistung) verabschiedet, die (mögliche) Anwendbarkeit der entsprechenden Praxis aber äusserst stark eingeschränkt: Solange jede einzelne Leistung den Leistungsvoraussetzungen genügt, ist sie zu vergüten, selbst wenn sich die Gesamtkosten einer Behandlung zu einer sehr hohen Gesamtsumme addieren. Eine absolute Obergrenze für die zulasten der obligatorischen Krankenpflegeversicherung gehenden Kosten besteht nicht, weder auf der Grundlage des Gesetzes noch aufgrund der bisherigen bundesgerichtlichen Rechtsprechung.

23 Diese Klarstellung löst das Problem der steigenden Gesundheitskosten nicht; es erhöht aber die Rechtssicherheit für alle Versicherten und Leistungserbringer, die nun (wieder) sicher mit der Vergütung ihrer Leistungen rechnen können. Gleichzeitig nimmt der Entscheid der Politik die Hoffnung, dass die Versicherungs- oder Gerichtspraxis das gesellschaftliche Grossproblem der steigenden Gesundheitskosten bewältigen wird, ohne dass ein politischer Preis (in der Form unpopulärer Entscheide) dafür zu zahlen ist.

24 Gefordert ist nun definitiv die Politik und damit die Gesetzgebung. Sie muss demokratisch legitimiert entscheiden, ob es überhaupt Kostenobergrenzen geben 
kann oder geben soll. ${ }^{14}$ Einem «bedside rationing» im Einzelfall hat das Bundesgericht mit dem vorliegenden Entscheid jedenfalls eine klare Absage erteilt.

$14 \overline{\text { Vgl. Bernhard Rütsche, Rechtsstaatliche Grenzen }}$ von Rationierungen im Gesundheitswesen, in: Ueli Kieser/Agnes Leu (Hrsg.), 5. St. Galler Gesundheits- und Pflegerechtstagung, St. Gallen 2018, $109 \mathrm{ff} ., 126 \mathrm{ff}$. 